\title{
A Standard Model Neutrino Mechanism
}

\author{
Robert B. Hayes \\ Nuclear Engineering Department, North Carolina State University, Raleigh, USA \\ Email: rbhayes@ncsu.edu
}

How to cite this paper: Hayes, R.B. (2021) A Standard Model Neutrino Mechanism. Journal of Modern Physics, 12, 1475-1482. https://doi.org/10.4236/jmp.2021.1211089

Received: July 26, 2021

Accepted: August 30, 2021

Published: September 2, 2021

Copyright (c) 2021 by author(s) and Scientific Research Publishing Inc. This work is licensed under the Creative Commons Attribution International License (CC BY 4.0).

http://creativecommons.org/licenses/by/4.0/

\begin{abstract}
This work argues a new standard model physics approach for neutrino oscillations by allowing neutrinos to have their flavor be entangled amongst all interacting fermions. Specifically, for a flavor conserved system, the effects from entanglement beginning at its origin and continuing through transit can give rise to the same observational outcomes as a flavor oscillation described by mass eigenstates. The implication being that although neutrino flavor is conserved in weak processes, this is argued to hold for all subsequent interactions. In so doing, the conventional neutrino mass propagator is argued to be a dimensional artifact of the oscillation being dependent on the linear density of material along the neutrino trajectory.
\end{abstract}

\section{Keywords}

Standard Model, Neutrino, Oscillation, Entanglement

\section{Introduction}

The existence of experimentally measured neutrino oscillations [1] has resulted in a consensus that neutrinos cannot have a zero rest mass [2] [3] giving rise to the expectation that new physics outside the standard model must be real. This is not unreasonable given that if a neutrino is traveling at light speed (and so massless), special relativity requires that its time will stand still preventing internal temporal changes (such as a flavor oscillation). If the neutrino does not travel at the speed of light, its spin could be reversed to an observer traveling faster than it and so realize a violation of spin conservation compounding the appeal to new physics. Given this, multiple models for neutrino oscillations have been proposed such as faster than light [4], sterile neutrinos [5] as well as other explanatory theories, all postulating physics outside the standard model [6] [7].

Still, quantum electrodynamics does allow particles traveling at light speed to become entangled throughout their existence. The initial postulate of the neu- 
trino existence was itself just a means to attempt justification of retaining energy conservation in beta decay [8]. Similarly, the initial proposition of color charge was a means to justify consistency of the Pauli exclusion principle for otherwise identical quark states [8]. With such inspiration, a mechanism is proposed to maintain the standard model of the neutrino by assuming the neutrino is simply entangled with other fermions throughout its interaction history giving rise to observed neutrino oscillations through entanglement.

The conventional new physics model for neutrino oscillations can be reduced to the form [9] $P_{v_{\alpha} \rightarrow v_{\beta}}=\sum_{k l}\left\langle v_{\beta} \mid v_{k}\right\rangle\left\langle v_{k} \mid v_{\alpha}\right\rangle\left\langle v_{l} \mid v_{\beta}\right\rangle\left\langle v_{\alpha} \mid v_{l}\right\rangle \mathrm{e}^{-i \frac{\left(m_{k}-m_{l}\right)}{\gamma} t}$. Here, $\alpha, \beta \in\{e, \mu, \tau\}, \alpha \neq \beta$, where the wavefunction is given by $\left|\Psi_{v}(t)\right\rangle=C_{e}(t)\left|v_{e}\right\rangle+C_{\mu}(t)\left|v_{\mu}\right\rangle+C_{\tau}(t)\left|v_{\tau}\right\rangle$ with the individual component amplitudes given by $C_{\xi}(t)=\sum_{j}\left[\sum_{k} \mathrm{e}^{-i m_{k} t} U_{\xi, k} U_{j k}^{*}\right] C_{j}(0)$. The unitary matrices for 2 flavors being simply $\boldsymbol{U}=\left(\begin{array}{cc}\cos \theta & \sin \theta \\ -\sin \theta & \cos \theta\end{array}\right)$. In the 3 flavor consideration, the mixing matrix can then be approximated (at small mixing angles $\theta_{j k}$ ) from the PMNS model as $\boldsymbol{U}_{v}=\left(\begin{array}{ccc}1 & s_{12} & s_{13} \mathrm{e}^{-i \delta} \\ -s_{12} & 1 & s_{23} \\ -s_{13} \mathrm{e}^{-i \delta} & -s_{23} & 1\end{array}\right)+O\left(s_{j k}^{2}\right)$ where $s_{j k}=\sin \theta_{j k}$ and and $U_{j k} \rightarrow\left\lfloor U_{j k}\right\rfloor \mathrm{e}^{-i \delta_{j k}}$ for the imaginary portions. The mass terms $m_{k}$ appearing in the $C_{\alpha}(t)$ amplitudes are invariably ascribed to the neutrino rest mass energy rather than being viewed as a phase parameter representing something else. This paper explores the possibility that the mass terms relate to the properties of the combined system of the neutrino with its history in such a way that the neutrino remains massless with the flavor variations being shared among the associated historically interacting fermions with that neutrino which force the observed oscillatory changes in the measurement via entanglement.

Others have considered the contribution of entanglement to the neutrino oscillation effect [10] [11] [12] [13] [14]. In such cases, these have consistently assumed the mass eigenstates as an accepted upset to the standard model for neutrinos. As such, the use of entanglement to help explain neutrino oscillations is not new. The current work tenets that mass eigenstates are not actually present, rather that the linear density of flavored material traversed by the neutrino is proportional to the probability of a flavor change due to ensuing entangling interactions.

\section{Theory}

A familiar means to create neutrinos using the standard Fermi diagrams is shown in Figure 1 (with pion decay in Figure 1(a) and muon decay in Figure (b)). Here the pion or muon would have originated in a proton collision interaction for accelerator based oscillation experiments. Neutrino chirality from the standard model had placed all $v_{\alpha}$ as left handed with $\bar{v}_{\alpha}$ being right handed 


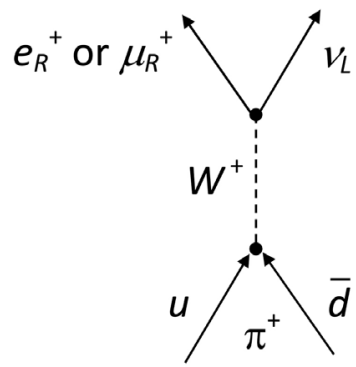

(a)

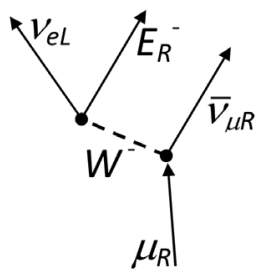

(b)

Figure 1. Feynman diagram for a characteristic pion decay followed by a potential muon creation and its decay. (a) on the left shows the initial pion decay with a muon decay (b) on the right.

[9]. Adding to this, the recognition that modern flavor dynamics require particle mass terms in the wavefunction definition, the existence of flavor superposition in the mass eigenstates has historically simply been assumed rather than allowed to arise from an entanglement response.

The key tenet proposed here is that in measuring the flavor of any one neutrino, this drives compensatory flavor changes in the associated particles coming out of the various generation processes from which it originated and may have subsequently become entangled, a neutrino flavor conservation. The neutrino flavor is then initially carried by the pion or muon in Figure 1 respectively allowing transfer to the neutrino which is entangled with the initial interaction particles creating the pion or muon (e.g., protons, neutrons, etc.).

Here, the initial proton or neutron wavefunction which gave rise to the eventual neutrino birth is then represented by $\left|\Psi_{p, n}(t)\right\rangle=\left(C_{e}(t)+C_{\mu}(t)+C_{\tau}(t)\right)|p, n\rangle$. The entanglement requirement can then be imposed that $\left\langle\Psi_{v}(t) \mid \Psi_{n, p}(t)\right\rangle=\langle v \mid n, p\rangle$ where $n, p$ is simply the neutron or proton originator. The oscillations are no longer a time dependent value when both are measured if no subsequent interactions had taken place.

As another example, standard positron decay in nuclear fusion as shown in Figure 2(a) would have its $v_{e L}$ flavor be entangled with the down quark in the neutron from which the $\mathrm{W}^{+}$boson interacted. This will require that if the electron interacts with a pion, it can take on some muonic flavor through entanglement which has a commensurate effect on the neutrino with which it is also entangled.

This particular scenario would require the probability of flavor transition for the down quark in the neutron to follow $P_{n_{\beta} \rightarrow n_{e}}=P_{v_{e} \rightarrow v_{\beta}}$ which then defines the initial flavor of the interacting down quark where $\beta \in\{\mu, \tau\}$. If such a neutron were found to decay after creation in an accelerator, the emergent proton and antineutrino would again share the flavor changes so that all particles would have to be measured to test flavor conservation for the reaction in Figure 2(a) and $P_{\bar{v}_{e} \rightarrow \bar{v}_{\beta}}=P_{p_{\beta} \rightarrow p_{e}}$ for the reaction in Figure 2(b).

Alternatively, if the antineutrino from Figure 2(b) were measured, the probability for the proton flavor could be probed to again test only $P_{\bar{v}_{e} \rightarrow \bar{v}_{\beta}}=P_{p_{\beta} \rightarrow p_{e}}$. 


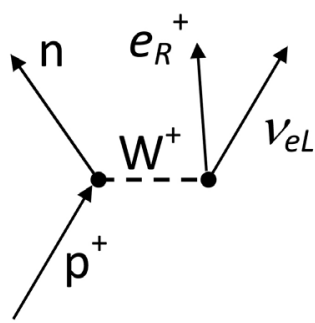

(a)

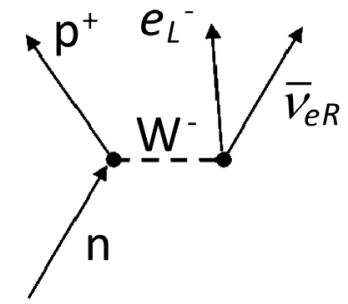

(b)

Figure 2. (a) (left) shows the conversion of an up to a down quark (requisite for hydrogen fusion into deuterium). (b) (right) shows basic neutron beta decay.

In short, an essential test of temporal neutrino flavor conservation will be a flavor measurement of all emanating particles at simultaneous delay periods (or prior to subsequent interaction with other flavored particles).

Given the entanglement condition upon creation, subsequent interactions will expectedly also allow additional entanglement with any and all Raleigh type neutrino scatters. All of these would then be proportional to the linear density $\rho_{L}$ of material along the neutrino path. The relative fractions of terrestrial electron flavor from radioactive decay of the primordial actinides along with historical mixing from cosmic and solar rays will then describe the relative densities for these entangled flavors. The density can then be broken up into the three orthogonal flavor components $\rho_{L}=\rho_{L e}|e\rangle+\rho_{L \mu}|\mu\rangle+\rho_{L \tau}|\tau\rangle$. The interaction probability $\sigma_{e, \mu, \tau}$ for a neutrino of one flavor to interact (becoming entangled) with a fermion having another flavor distribution would not necessarily be equivalent and so is left symbolically for now and is assumed to be a measurable quantity as described above.

The individual elements can then be written as $\sigma=\left(\begin{array}{ccc}1 & \sigma_{\mu e} & \sigma_{\tau e} \\ \sigma_{e \mu} & 1 & \sigma_{\tau \mu} \\ \sigma_{e \tau} & \sigma_{\mu \tau} & 1\end{array}\right)$. Specifically, there will be a proportionality between the traditional mixing matrix for the "new physics" mass eigenstates $U_{\alpha j}$ with both the fermionic linear densities $\rho_{L}$ and the interaction probability of the form $U_{i j}=\sigma_{i k} \delta_{k j} \rho_{k}$ As such, the propagation for the standard model neutrino does not oscillate in the traditional sense but rather undergoes entanglement via interaction with other fermions (including primordials).

The currently measured values for the $U_{i j}$ elements are associated with the new physics oscillation probabilities given by [8] $P_{\nu_{\mu} \rightarrow v_{e}}=\sin ^{2} 2 \theta \sin ^{2} \frac{\Delta m^{2} L}{4 E_{0}}$, where the probability of flavor transition in the left handed electron neutrino coming from the same decay process would then be approximated by $P_{v_{e} \rightarrow v_{\beta}}=1-P_{v_{\mu} \rightarrow v_{e}}-P_{v_{\mu} \rightarrow v_{\tau}}$. Here, the mass term $m$ used in that probability estimate has been associated with the quantum mechanical oscillation property of standard particles being left handed excluding the Dirac mass Lagrangian [15] 
$\mathcal{L}^{D}=\sum_{\alpha, \beta=e, \mu, \tau} \bar{v}_{\alpha L} M_{\alpha \beta}^{D} v_{\beta R}$. Rather, even when accepting only the Majorana version [16] $\mathcal{L}^{M}=-0.5 \sum_{\alpha, \beta=e, \mu, \tau} \bar{v}_{\alpha L} M_{\alpha \beta}^{M} v_{\beta L}$ the requisite mass term remains. Here, the mass terms for the various flavor oscillations $M_{\alpha \beta}$, are assumed nonzero from which an isolated neutrino then has a time dependence of $\left|v_{\alpha}(t)\right\rangle=\mathrm{e}^{-i m_{\alpha} t}\left|v_{\alpha}(0)\right\rangle$ using the standard convention of $c=\hbar=1$. That the propagator in these units has dimensionality of mass does not require that the neutrinos have mass a priori when the matrix $M_{\alpha \beta}$ is effectively just a fit to the measurement data. The implication being that the mass corresponds to $\rho_{L}$ and forces a neutron oscillation through entanglement only. This then allows all neutrinos to remain left handed and so remain consistent with the standard model [9].

It might be helpful to consider this alternative view by casting the mass terms from the new physics model in units of $\mathrm{eV} \mathrm{c}^{-2}$, then for neutrino phase equivalent estimates of [17] $0.071,0.072$ and $0.087 \mathrm{eV}$, this would allow us to write $C_{\xi}(t)=\sum_{j}\left(\mathrm{e}^{-i 0.071 t} U_{\xi, 1} U_{j, 1}^{*}+\mathrm{e}^{-i 0.072 t} U_{\xi, 2} U_{j, 2}^{*}+\mathrm{e}^{-i 0.087 t} U_{\xi, 3} U_{j, 3}^{*}\right) C_{j}(0)$. Again, this in no way is evidence for or against a neutrino mass but acknowledges the observational outcomes where the linear energy density of the neutrino history is predicted to give rise to the phase estimates [17]. Without contradictory evidence, the use of $U_{i j}=\sigma_{i k} \delta_{k j} \rho_{k}$ appears to be fully consistent with the observation of neutrino flavor variation during transport. The model will also be entirely falsifiable or verifiable when historical values of $\rho_{L}$ are considered in neutrino oscillation measurements.

\section{Test Options}

With the decay scheme in Figure 1, all emanating neutrinos will conserve flavor, collectively with their initiating nucleon. This offers a test mechanism but does require correlating each emergent neutrino along with its creation ejecta. Reactor and solar neutrinos are not operationally practical to track their progenitor dynamics outside of expected average values for $\rho_{L}$ but accelerator neutrinos may allow a more practical method for testing this theory. This may prove to be the most economical way but would require the substantial effort to calculate the $\rho_{L}$ for these dynamic historical measurements (geoneutrinos, solar neutrinos etc.) with the largest expected uncertainty coming from interactions with the remnant primordial fermion distributions.

The proton beams from the T2K [18], NOvA [19] and CERN [20] could all be modified to track the initial collision excreta although probing these for neutrino flavor which would require a secondary neutrino beam to collect appropriate interaction data. Clearly this also is not a trivial task but in the end, it could provide a means to allow neutrinos to remain in the standard model and still explain all observational measurement results.

\section{Discussion}

One can insist on a continuous, deterministic physical interpretation of what is 
happening to the neutrino during transport prior to measurement allowing a predictable oscillation behavior but the alternative answer offered here is the same evolution observations being driven by entanglement. Examples of comparable deterministic looking physics with a quantum mechanism include magnetic resonance or the familiar 2 slit experiment. In the resonance behavior of electron paramagnetic or nuclear magnetic resonance, the fermion spin is actually in only ever one of two states (parallel or anti parallel to the external magnetic field) but the superposition of both states allow a traditional precession based resonance effect following classical rules as a statistical average. With the two slit experiment, the wavefunction transports through the penetrations as a pure probability density function (a superposition of all possible states) obeying only wave mechanics during transmission requiring interference opportunities until measured post transit.

The new physics derivation of neutrino oscillations assumes a right handed neutrino to exist giving rise to the Dirac mass term. That the derivation gives the right answer, does not necessarily require the interpretation of that derivation to be correct (similar to the Bohr model of the hydrogen atom) and is in the end a fitted result to measurement data. Rather, the argument offered here is that the probability evolution of the neutrino is constrained by its being entangled from its evolution enabling flavor conservation. Here, all associated ejecta from the origin are all evolving through time which then forces the neutrino to likewise evolve to maintain the standard model conservation laws. Those particles which do not carry flavor then are unable to oscillate providing said test of the theory.

More importantly, for any given neutrino source and associated detector, the fermionic density will not be the same. These will depend on both the source (solar, reactor, primordial, radioactivity and accelerator) and detector locations. Estimations of the current entangled distribution in each source detector line segment would have to be done on a case by case basis but is within the realm of reason and not carried out further here. In this sense, the transition probabilities

$P_{v_{\alpha} \rightarrow v_{\beta}}$ for a given measurement result could be calculated from base principles rather than being fit to the data.

\section{Conclusion}

A new theory has been put forward which allows neutrinos to effectively oscillate using only standard model physics where neutrino flavor is still conserved. Effectively, the individual neutrino flavor changes are related back to being entangled with the combined system from which the neutrino originated (e.g., scattering and decay process, etc.). The flavor changes taking place in all associated particles emanating from its evolution starting with its last interaction then becomes an experimentally testable feature of this model which would in principle, explain neutrino oscillations without a requirement for any new physics. Tests could be comprised of measuring all flavors of excreta products and paths to demonstrate the conservation of lepton flavor and parity expectation from the 
standard model.

\section{Acknowledgements}

This material is based upon work supported by the Department of Energy National Nuclear Security Administration under Award number DE-NA0002576. Additional support of this work was through a joint faculty appointment between North Carolina State University and Oak Ridge National Laboratory. The data that supports the findings of this study are available within the article.

\section{Conflicts of Interest}

The author declares no conflicts of interest regarding the publication of this paper.

\section{References}

[1] Pallavicini, M. (2015) Journal of Physics. Conference Series, 598, Article ID: 012007. https://doi.org/10.1088/1742-6596/598/1/012007

[2] Dragoun, O. (2018) AIP Conference Proceedings, 1686, Article ID: 020008.

[3] Fertl, M. (2018) Hyperfine Interactions, 259, 1-6. https://doi.org/10.1007/s10751-018-1530-2

[4] Ehrlich, R. (2019) Advances in Astronomy, 2019, Article ID: 2820492. https://doi.org/10.1155/2019/2820492

[5] Kisslinger, L.S. (2016) International Journal of Modern Physics, 31, Article ID: 1630037. https://doi.org/10.1142/S0217751X16300374

[6] Cai, Y., Herrero, G.J., Schmidt, M.A., Vicente, A. and Volkas, R.R. (2017) Frontiers in Physics, 5, 63. https://doi.org/10.3389/fphy.2017.00063

[7] Studenikin, A.I. (2016) Nuclear and Particle Physics Proceedings, 273-274, 1711-1718. https://doi.org/10.1016/j.nuclphysbps.2015.09.276

[8] Griffiths, D. (1987) Introduction to Elementary Particles. John Wiley \& Sons Inc., New York. https://doi.org/10.1002/9783527618460

[9] Suekane, F. (2015) Neutrino Oscillations: A Practical Guide to Basics and Applications. Springer, New York. https://doi.org/10.1007/978-4-431-55462-2_4

[10] Nauenberg, M. (1999) Physics Letters B, 447, 23-30. https://doi.org/10.1016/S0370-2693(98)01556-1

[11] Akhmedov, E.K. and Smirnov, A.Y. (2011) Foundations of Physics, 41, 1279-1306. https://doi.org/10.1007/s10701-011-9545-4

[12] Cohen, G.C., Glashow, S.L. and Ligeti, Z. (2009) Physics Letters B, 678, 191-196. https://doi.org/10.1016/j.physletb.2009.06.020

[13] Wu, J., Hutasoit, J.A., Boyanovsky, D. and Holman, R. (2011) International Journal of Modern Physics A, 26, 5261-5397. https://doi.org/10.1142/S0217751X11054954

[14] Kayser, B., Kopp, J., Robertson, R.H. and Vogel, P. (2010) Physical Review D, 82, Article ID: 093003. https://doi.org/10.1103/PhysRevD.82.093003

[15] Verma, S. (2015) Advances in High Energy Physics, 2015, Article ID: 385968. https://doi.org/10.1155/2015/385968

[16] Bilenky, S.M. (2004) Proceedings of the Royal Society of London, 460, 403-443. https://doi.org/10.1098/rspa.2003.1263 
[17] Singh, M. (2020) EPL (Europhysics Letters), 129, Article No. 11002. https://doi.org/10.1209/0295-5075/129/11002

[18] Abe, K., Abgrall, N., Aihara, H., Ajima, Y., Albert, J.B., Allan, D., Amaudruz, P.A., Andreopoulos, C., Andrieu, B., Anerella, M.D. and Angelsen, C. (2011) Nuclear Instruments and Methods in Physics Research Section A: Accelerators, Spectrometers, Detectors and Associated Equipment, 659, 106-135.

[19] Ahn, M.H., Aliu, E., Andringa, S., Aoki, S., Aoyama, Y., Argyriades, J., Asakura, K., Ashie, R., Berghaus, F., Berns, H.G. and Bhang, H. (2006) Physical Review D, 74, Article ID: 072003.

[20] Adam, T., Agafonova, N., Aleksandrov, A., Altinok, O., Sanchez, P.A., Anokhina, A., Aoki, S., Ariga, A., Ariga, T., Autiero, D. and Badertscher, A. (2012) Journal of High Energy Physics, 2012, 93. 